Cómo citar: J. Rivera, “Editorial. Entre el recurso hídrico y el medio ambiente. Consideraciones frente a los Objetivos del Milenio", Inventum, vol. 15, no. 29, pp. 1-2. doi: 10.26620/uniminuto.inventum.15.19.2020.1-2

Editorial: Corporación Universitaria Minuto de Dios - UNIMINUTO.

ISSN: $1909-2520$

elSSN: $2590-8219$

Conflicto de intereses: los autores han declarado que no existen intereses en competencia.
EDITORIAL

\section{ENTRE EL RECURSO HÍDRICO Y EL MEDIO AMBIENTE. CONSIDERACIONES FRENTE A LOS OBJETIVOS DEL MILENIO}

El número 29 de la revista Inventum de la Facultad de Ingeniería de la Corporación Universitaria Minuto de Dios (UNIMINUTO), presenta siete artículos externos de carácter nacional y uno de carácter internacional, los cuales reflejan la inclusión de las ingenierías y las tecnologías con los procesos tecnológicos en donde cada uno de ellos contribuye desde la experticia de los autores a la divulgación de los resultados de investigación adelantados en diferentes comunidades tanto rurales como municipales, lo cual se traduce en el enfoque de esta publicación enfocada en el hábitat, productividad, ciencias básicas y la convergencia digital.

Dentro de los artículos publicados, se hace alusión al recurso agua y al desarrollo e implementación de tecnologías para su mejor utilización y aprovechamiento. Dentro del primer artículo, con la utilización de los recursos maderables del medio ambiente, se propende por la maximización de la recarga de agua subterránea. En el segundo artículo, los autores elaboran un diseño hidrológico bajo modelos matemáticos que permiten la producción agroecológica desde un agroecosistema integrado de cultivos y mediante Sistemas de Información Geográfica (SIG). Así mismo, en otro artículo se aborda la implementación de dos metodologías conjuntas con el fin de obtener una mayor eficiencia en la optimización de captación de agua subterránea como sistemas de producción, lo cual se corrobora mediante una comparación entre los litros de agua que se obtienen en cada caso.

Dentro de esta producción, se hace énfasis en la importancia del medio ambiente, ya que en uno de los artículos se presenta la elaboración de una guía metodológica para la caracterización y cuantificación frente al manejo de los residuos teniendo en cuenta el resultado de la producción per cápita obtenida por cálculos, lo cual debe coincidir con la (ppc) del estrato real en donde se esté trabajando, lo que permite confirmar que la cuantificación estuvo bien realizada y por consiguiente la caracterización es adecuada.

Se genera también un aporte significativo centrado en el desarrollo empresarial, cultural, ambiental y tecnológico que permea sobre el proceso educativo, y finalmente, se contribuye al desarrollo de un análisis y modelado de los resultados obtenidos en cuanto a la medición de material particulado, con el fin de identificar los niveles de riesgo a los cuales están expuestos los ciclistas en su proceso de movilidad hacia la universidad 
sobre la ruta perteneciente a la localidad de Engativá, de la cual no se tienen estudios de material particulado ni mediciones previas de este tipo de partículas.

Finalmente, aparecen reflejados los resultados de investigación de dos publicaciones centradas en comunidades: una de ellas para el sector vehicular, que propende por la minimización de los niveles de riesgo en carreteras topográficas y los niveles de accidentalidad con la predicción de un modelo matemático. De otra parte, el otro artículo se centra en procesos se simulación discreta para una unidad de quimioterapia en cuanto al flujo de pacientes, con el fin de mejorar la utilización de los recursos disponibles mediante diferentes variables clave, como el arribo de pacientes, el tiempo de estadía, personal requerido y las operaciones asociadas para lo cual se propone una metodología de segmentación de pacientes con base en los tiempos de infusión según protocolos. Lo anterior refleja los productos de investigación derivados desde una perspectiva holística, para el desarrollo de la ciencia, la ingeniería y las tecnologías, teniendo en cuenta la interdisciplinariedad de diferentes saberes.

Julio César Rivera Rodríguez

Editor 